Full citation reference for this paper: EVETT, L., BATTERSBY, S., RIDLEY, A. and BROWN, DJ., 2009. An interface to virtual environments for people who are blind using Wii technology - mental models and navigation . Journal of Assistive Technologies. vol 3 (2), pp. 30-39.

\title{
An interface to virtual environments for people who are blind using Wii technology - mental models and navigation
}

\author{
Lindsay Evett*, Steven Battersby, Allan Ridley and David Brown \\ Interactive Systems Research Group \\ Computing and Technology \\ Computing and Informatics Building \\ Nottingham Trent University \\ Clifton Lane \\ Clifton \\ Nottingham NG11 8NS \\ UK \\ lindsay.evett@ntu.ac.uk, superski@sky.com, \\ steven.battersby@ntu.ac.uk, david.brown@ntu.ac.uk
}

*To whom correspondence should be addressed

\section{Brief biography for Dr Lindsay Evett}

Lindsay is a lecturer in the Computing \& Technology Team. Her research is on accessibility and assistive technology, especially with respect to Serious Games, and webbased content. She is a lecturer in Artificial Intelligence, and a member of Nottingham Trent University's working group on accessibility. She is a co-investigator on the GOET European project on serious educational games to develop prevocational skills in people with learning difficulties.

\section{Brief biography for Steven Battersby}

Steven is a software design engineer for the Interactive Systems Research group and has worked on numerous projects concerned with Serious Games and assistive technology. Steven is currently completing a $\mathrm{PhD}$ on adaptive, assistive technology.

\section{Brief biography for Allan Ridley}

Allan was recently awarded an MRes with distinction in Computer Science by Nottingham Trent University. He is just starting a $\mathrm{PhD}$ on accessible interactive systems. He has worked as an assistive technology trainer.

\section{Brief biography for Professor David Brown}

David was promoted from Reader to Professor of Interactive Systems for Social Inclusion in 2007. His research focuses on the application of virtual environments for the education of people with an intellectual impairment and for rehabilitation. His research on virtual environments for people with learning disabilities has been funded by a range of government agencies, by EPSRC and the EU. He is consortium leader for "Game on", to develop 3D role play games for the education and personal development of prisoners and those at risk of offending. He is the principle investigator for the GOET European project 
on serious educational games to develop prevocational skills in people with learning difficulties.

Acknowledgements: This underlying research in games supports EU Leonardo Project GOAL.NET (UK/07/LLP-LdV/TOI-009) 


\title{
An interface to virtual environments for people who are blind using Wii technology - mental models and navigation
}

\begin{abstract}
Accessible games, both for serious and for entertainment purposes, would allow inclusion and participation for those with disabilities. Research into the development of accessible games, and accessible virtual environments, is discussed. Research into accessible Virtual Environments has demonstrated great potential for allowing people who are blind to explore new spaces, reducing their reliance on guides, and aiding development of more efficient spatial maps and strategies. Importantly, Lahav and Mioduser $(2005,2008)$ have demonstrated that, when exploring virtual spaces, people who are blind use more and different strategies than when exploring real physical spaces, and develop relatively accurate spatial representations of them. The present paper describes the design, development and evaluation of a system in which a virtual environment may be explored by people who are blind using Nintendo Wii devices, with auditory and haptic feedback. The nature of the various types of feedback is considered, with the aim of creating an intuitive and usable system. Using Wii technology has many advantages, not least of which are that it is mainstream, readily available and cheap. The potential of the system for exploration and navigation is demonstrated. Results strongly support the possibilities of the system for facilitating and supporting the construction of cognitive maps and spatial strategies. Intelligent support is discussed. Systems such as the present one will facilitate the development of accessible games, and thus enable Universal Design and accessible interactive technology to become more accepted and widespread.
\end{abstract}

Keywords: accessible games; accessible virtual environments; cognitive maps; Design for All; Serious Games; Universal Design, Wiimote; WWii 


\section{An interface to virtual environments for people who are blind using Wii technology - mental models and navigation}

\section{INTRODUCTION}

Terraformers is a visual/audio hybrid navigation quest game, which is playable by both sighted players and by players who are visually impaired and/or blind (Westin, 2004; PIN, 2005). The aim of the game is to find parts of a computer, which would help defeat some evil robots. Terraformers offers auditory navigation and game information, which blind players can use successfully to navigate its virtual environment and to play the game. Previous auditory games have been created without graphics, meaning that they are unsuitable for sighted players. Such games do not encourage integration; Terraformers includes graphics (which can be switched off) so that blind and sighted players can play together. Such accessible games are a great step forward in creating accessible and inclusive systems. The team at ICS-FORTH has developed a design methodology for developing Universally Accessible games (UA-Games, Grammenos et al, 2005). Instead of either producing games which are largely inaccessible to anyone with a disability, or games which can only be played by people with a particular disability (such as audio games for the blind, single switch games for the motor impaired) this approach aims to produce games which have high interaction quality, and are concurrently accessible to people with diverse abilities. UA-Games follow the principles of Design for All (or Universal Design) and are designed for, and to adapt to, different individual characteristics without the need for further adjustments. Currently, the team at ICS-FORTH has developed two games: UA-Chess, a universally accessible web-based chess, and Access Invaders, a universally accessible multiplayer/multiplatform version of Space Invaders (Grammenos and Savidis, 2006; and see McCrindle and Symons, 2000). 
The design process followed at ICS-FORTH for the creation of UA-Games consists of two key stages. Initially, abstract task definition specifies the interactive game space at an abstract level, without reference to the physical-level of interaction (e.g. input/output devices, rendering). The next stage specifies the lower-level design details, incrementally moving towards the physical level of interaction by addressing particular user characteristics. The direct involvement of representative end-users with diverse characteristics, and domain experts, is promoted so that the design outcomes are continuously assessed. Aspects of the design may be revisited on the basis of this assessment.

Research into navigating around virtual worlds by people who are blind has shown that Virtual Environments (VEs) can support and facilitate their construction of cognitive maps. People who are blind tend to adopt sequential, route-based strategies for moving around the real world; common strategies take the self (or body) as the main frame of reference, as opposed to an external frame of reference (e.g., see Golledge et al, 1996). There is a great deal of variability in the navigational strategies used by people who are blind, with those who perform better in navigational tasks being those who use more map-based strategies (Hill et al, 1993). Training in such strategies can greatly improve performance (Cummins and Rieser, 2008; Simonnet et al, 2006). Simonnet et al (2006) have detailed documented strategies and distinguish between egocentric frames of reference, where objects and locations are related to a person's particular perspective, and exocentric frames of reference, where external frames of reference are used. External frames of reference and map-based strategies allow more efficient representation which enables flexibility, so that alternative routes can be taken, shortcuts can be made and destinations changed, because they encompass a more complete representation of the environment to enable navigation 
from a number of perspectives (Golledge et al, 1996). The actual mobility training people who are blind receive is generally basic and egocentric (see section 3.2.2 below).

VEs have great potential for supporting and facilitating the construction of exocentric cognitive maps by those who are blind. The visual modality lends itself to global spatial representations. These are more difficult to derive from haptic (i.e., relating to the sense of touch) sources, which are by their nature more local and sequential. These properties of haptic information, combined with the fear of collisions, encourage the use of egocentric frames of reference by people who are blind. However, functional equivalence of haptic and other non-visual information to visual information can, under the right conditions, be indicated and the possibility that they may all contribute to amodal spatial representations exists (Loomis and Klatzky, 2008). Cognitive maps can be created by the blind; it has already been noted that the more successful navigators tend to use more map-based strategies. Lahav and Mioduser $(2005,2008)$ demonstrated that interacting with a haptic virtual environment (using haptic and auditory feedback) can facilitate the construction of mental maps of spaces and thereby contribute to blind people's spatial performance. They found that, when an experimental group of people who are blind explored a haptic virtual environment, they developed new strategies, and applied existing strategies for navigation in different ways, compared to a control group, who explored the real physical environment. When questioned about the environment, the experimental group had better structural descriptions of it and the location of objects within it. Further, when the experimental group went on to explore the real environment, they were more successful in completing target and perspective oriented tasks, and completed them more quickly using more direct routes than the control group. These results strongly suggest that the experimental group had developed cognitive maps with properties much more like those of 
sighted navigators. That is, exploring the VE and experiencing the associated feedback appeared to enable the development of a more complex, wholistic, exocentric cognitive map of the environment.

There is research on usability analysis of sound-based virtual environments and spatial sound (Sanchez et al, 2002). Today, most audio games are oriented towards functionality, and encourage players to understand the games space by using three listening modes (Chion, 1994). Casual listening is the most common, and requires the listener to attune to the source or origin of the sound. An example is the recognition of a person by their voice. Semantic listening is the understanding of voice or language. This is the most complex of listening modes and requires the most attention. Reduced listening focuses on the parts of a sound (e.g., the pitch, tone or footsteps). These listening modes are all functional for audio game players and have varying attentional requirements.

Research on the utility of haptic feedback, often using the PHANToM force feedback device, has demonstrated its effectiveness (see Lahav and Mioduser $(2005,2008)$ above, also Petrie et al, 2000). However, the PHANToM device is very expensive, well beyond the means of ordinary people.

Choosing sound feedback sensitively, and incorporating other types of feedback, can result in usable and effective interactive systems for those who are blind. Being able to experience and practice various skills by interacting with a VE (especially navigation), and being able to interact with VEs and the various types of games which use them (both Serious games and those for entertainment) has great potential for people who are blind. 
The present paper describes the research into and development of a virtual reality interface for people who are blind, which uses a mix of modalities for feedback. Unlike some systems, this system uses contemporary gaming technology which is readily available, and cheap. The present research has investigated the use of the Nintendo Wii remote controller (Wiimote) as an adaptive assistive device, and in this case as a device for people who are blind to use for interacting with virtual environments. The Wiimote allows the user to interact with a system via movement and pointing. In addition, visual, auditory and haptic feedback is available. Using the Wiimote has many advantages, not least that it is mainstream, easily available and relatively cheap.

\section{THE WIIMOTE AS AN ASSISTIVE DEVICE}

The Wii has been investigated as a platform for the development of assistive technology, by interfacing the Wiimote with a personal computer, and by detailing the technologies involved (Battersby, 2007; 2008).

\subsection{Interfacing a Wiimote with a PC}

Using the Wiimote's Bluetooth capability it proved to be a simple task to interface the Wiimote with a standard PC. The Windows Wii application (WWii) was created to handle connection and pairing between the Wiimote and the target operating system. Following on from earlier iterations (see Battersby, 2008), WWii has been used to facilitate further research and development. WWii is a functional windows-based driver application written in C\# with Peek's (2008) Managed Library for Nintendo's Wii remote (Wiimote) as its heart. It provides facilities for keyboard, mouse and joystick mapping, for peripheral input 
data capture and a basic capacity for the replay and analysis of any captured data. WWii not only provides the facility for the Wiimote to be mapped to the windows system but also supports multiple extensions such as the Wii Classic Controller, the Wii Nunchuck and the Wii Fit board. Interface panels have been created to support mapping functionality by enabling a user to configure each of the Wiimote's physical inputs to any desired keyboard or mouse input combination (see figure 1). In effect this enables the Wiimote to be seen by the system as any of the highlighted devices, limited only by the volume of inputs available from the device. Customizable feedback is developed through the feedback mapping panel, where flags may be set to operate any of the Wiimote's feedback mechanisms.

Figure 1 about here

2.2 Wiimote Sensor Technology

\subsubsection{The Accelerometer}

The accelerometer contains a micro mechanical structure supported by silicon springs. It measures linear accelerations along three fixed axes; it is unable to distinguish between linear motions and rotations, and so it is capable of measuring only pitch and roll angles.

\subsubsection{The Optical Sensor}

The optical sensor is an infrared camera situated at the front end of the Wiimote. The camera is connected to an integrated image analysis chip that can identify up to four 
individual Infrared light sources and report their position, approximate size and level of intensity. The light sources for the present application are provided in the form of two clusters of infrared LEDs situated at two opposite ends of a stationary bar, which was a modification of an existing USB/battery powered product. The image sensor sees the light as two bright dots separated by a known distance. Triangulation is used to calculate the distance between the bar and the Wiimote. The camera projects a $1024 \times 768$ plane in front of the user and positional/rotational data is obtained in reference to this plane. This is possible as the position of the sensor bar and distance between the LED clusters remains constant. This system allows the Wiimote to function as an accurate pointing device and provides the yaw value, so that the Wiimote can be tracked in 3D space.

\subsection{Practical Investigations of Wiimote Sensors}

In order to test the interface functionality of the two Wiimote sensors a simple test environment was created that consisted of a virtual representation of the Wiimote within 3D space. The environment was constructed within AutoDesk's 3D Studio Max application and interactive capability was provided by importing the environment into Adobe Director, where navigation and response to system input could then be coded. Successful performance was demonstrated by slaving the motion of the virtual Wiimote to that of a real world counterpart. The initial testing of the Wiimote sensors highlights the Wiimote and its technologies as more than adequate for the development of assistive concepts and solutions. Used in conjunction they provide the required 6 degrees of freedom needed to orientate a body within 3D space. The Wiimote can be used as a 2D pointing device via the optical sensor and can easily provide 2D mouse-like interaction. 


\subsection{Feedback functionality of the Wiimote}

In addition to providing system input the Wiimote is capable of providing basic auditory, visual and haptic feedback. The speaker on the Wiimote allows audio content to be transmitted from the host to be played back. By default the Wiimote indicates its player assignment by lighting the corresponding LED. The LEDs are also used to indicate the power level of the Wiimote's battery upon power up. This visual feedback is redundant for the present application. The Wiimote also includes a rumble device which can be set to either an on or off state. Manipulation of the frequency of operation can be used to provide pulses to create the illusion of differing strengths of rumble. This device offers great potential for haptic feedback.

\subsection{Expansion capabilities of the Wiimote}

The Wiimote features an expansion port. This provides the facility for the connection of additional auxiliary controllers to augment the input capabilities of the Wiimote. The Wiimote's assistive capabilities can also be expanded via implementation of software solutions.

All the functional attachments use the Bluetooth interface of the Wiimote to communicate with the Wii console, thus allowing them to be much simpler and cheaper to implement than the Wiimote itself. Attachments can be added and removed from the Wiimote whilst in use without resulting in device damage. The main attachment to date for the Wiimote is the Nunchuk controller. The primary component of the Nunchuk is an analogue 
thumbstick, which outputs 2D axis data. In addition to the thumbstick the Nunchuk has two buttons labeled as $\mathrm{C}$ and $\mathrm{Z}$ and a three-axis accelerometer sensor.

\section{THE WIIMOTE AS A CANE WITHIN 3D ENVIRONMENTS}

The Wiimote's ability to describe a body within 3D space means that it can be used rather like a cane, and therefore provide an interface to 3D environments for the visually impaired. The virtual Wiimote counterpart uses distance measurements obtained by ray casting to control feedback in the form of vibration and auditory signals.

\subsection{Design}

In order to investigate the potential of the Wiimote as a virtual cane a test environment was created. Additional functionality was added to the WWii enabling it to host Shockwave environments thus providing direct communication between the environment and the Wiimote. Other environments could easily be loaded into the application.

The design of the Wii cane system was informally evaluated and then modified; this occurred repeatedly in order to evolve a functional system. The modifications were carried out by the system design engineer (Battersby, 2007; 2008), who conducted the evaluations together with a Computer Science Masters degree student who is blind (subject A). This iterative design process was in line with user-centred design methodology (e.g., Lannen et al, 2002; Battersby et al, 2004). Early design iterations highlighted the need for initial orientation and calibration. A number of variables needed to be explored, particularly the relationship between the motion of the Wiimote and its motion relative to the environment, 
and the type and nature of the different types of feedback and their relationship to the environment and the objects in it. A number of alternative configurations were explored, leading to a design suitable for testing and evaluation. For all configurations, the Wiimote is used to scan the environment in front of the user; the whole plane can be scanned, that is left-right, up-down, or any other angle. Speed of motion in the VE was set at the speed identified as comfortable from extensive experience of user testing in Serious Games; strides were set at about a metre for the same reason (e.g., Brown et al, 2007).

\section{Configuration 1:}

The Nunchuck was used. The thumbstick could be used to direct motion in eight directions at each 45 degree point. The Nunchuck was used to determine direction with respect to self. That is, whatever direction the thumbstick was held in, when the $\mathrm{Z}$ button was pressed motion would be in that direction. The aim was to provide an on the spot point of selfreference. As with a person turning, the thumbstick would be turned and motion would proceed in that direction when the button was pressed. Rumble varied according to distance from an object, with constant rumble on collision. Pressing a button produced spoken distance and object name. However, there is no external frame of reference with respect to the environment, and the user was disoriented.

\section{Configuration 2:}

For configuration 2 the Nunchuck was removed because the added complexity appeared to outweigh any benefits. Configuration 1 needed additional environmental cues in order to orient the user within the space. Some available possible cues are footsteps, rumble on approach (pulsing could indicate distance), rumble on collision (constant), sonar and auditory signposts. The feedback used was rumble (constant) on collision, and sonar, 
whereby frequency of beeps indicated distance. As with the previous configurations, pressing a button produced spoken distance and object name. In addition, on entering a space, a description of the space is available via a button press. In order to turn, the Wiimote was rolled to the left or the right. A beep indicated turning mode, and every beep indicated a 10 degree turn. The left and right beeps were different to make them discriminable. Motion was provided by a button press on the Wiimote, inducing motion in the last facing direction. Because of the positions of the various buttons on the Wiimote, it proved difficult to control; additionally, button presses tended to move the Wiimote, producing positional confusion. The Nunchuck was therefore re-introduced in configuration 3 .

\section{Configuration 3:}

In this final configuration, left and right turning was achieved by rolling the Nunchuk to the left and to the right, producing a 15 degree turn. Left and right step sounds provided turning feedback, which proved easy to interpret. Motion forwards and backwards was initiated by tilting the Nunchuk up or down. The Wiimote was used for scanning the space. Beeps were used for indicating different types of objects and their distance. Different tones of beep were used to indicate furniture, walls, doors, floors and out of scanning plane (accompanied by a constant rumble). The rate of the beeps increased as any object was approached. There was a constant rumble on collision. Subjects were told when they had passed from one space to another (for the last subject this was implemented as a whooshing sound on transition). As with the previous configurations, pressing a button produced spoken distance and object name. 
This configuration separated motion (controlled by one hand with the Nunchuk) and scanning (controlled by the other hand with the Wiimote), and this is a clearer arrangement. The design now appeared to contain the necessary information for successful navigation, and was stable enough for evaluation.

\subsection{Evaluation}

\subsubsection{Test environment}

Having arrived at the system design of configuration 3, another VE was created for testing the system. This VE is a representation of part of the third floor of the Nottingham Trent University Computing and Informatics building (see Figure 2 for floor plan). This was chosen for a number of reasons:

1. It is easily available

2. It is a real functional space

3. It contains an open plan area with a number of obstacles of different types within it. Consequently it contains numerous potential areas for navigation and numerous potential navigation and perspective tasks

4. It is irregular, the level of complexity encouraging development of spatial awareness and navigational skill

Figure 2 about here 


\subsubsection{Subjects}

Subject A: subject A is 52. He is the person involved with the design of the system. He has no functional vision. He can under some circumstances detect light, although this has to be very bright. His eyes were not formed properly at birth. He has Hallerman-Streiffe syndrome. He was blind until about the age of 2 , when he had surgery to restore his sight. After the surgery he had functional sight; his distance vision was fine but close up he could not read without a lens, or recognise faces. He could move around and navigate without major difficulty. His sight began to deteriorate in his late 30 s until the age of 42 , when he had no useful remaining vision. He received basic training in the use of the cane around this time. This involved holding the cane and sweeping and turning, identifying landmarks, and the use of inner and outer shorelines. There was some discussion of using smells and sounds. There was some learning of routes. The training focused on egocentric and route strategies. Beyond this people develop their own strategies, and all three subjects reported doing so. Independent traveling in new spaces is very difficult; in such cases, a trainer will guide the person in at least the first instance. This is also true when dogs are used. The trainer will come out to help the person and the dog identify reference points for future use. Subject A has been guided by dogs for some years. Training with a dog is intense, starting with three weeks solid at a centre or at home. Routes are learnt by walking the route with the trainer, and identifying reference points to tell the dog to go to. Subject A is currently with his fourth dog, and so is experienced at being part of a dog/person team. He commonly travels alone with the dog and is a frequent user of public transport.

Of the three people who took part in this evaluation, subject A visits the space being used the most often. However, while he frequently follows a single route from the entrance to an 
office at the far end, most of the rest of the space is unknown to him, and this was confirmed during his exploration of the virtual version of it.

Subject E: subject E is 27 and is partially sighted, although could be registered blind if she requested it. Currently she has minimal vision in her left eye, which is only apparent when her right eye is shut or obscured. In the right eye she has tunnel vision, with a section missing on the nasal side, and blind spots. She was very short sighted, with some retinal damage, up until 9 years ago when she suffered several bouts of optic neuritis. Her vision is currently stable but likely to get worse. She received basic cane training, similar to that of subject A, when her vision first deteriorated. She is familiar with a limited part of the area being used in this study. That is, she often traverses a single route between two doors across the top of the space. As with subject A, the rest of the space is mostly unknown to her, again confirmed when she explored the virtual version of it.

Subject $\mathrm{H}$ : subject $\mathrm{H}$ is 38 , and was very short sighted up until the age of 17 , when he was registered blind. His sight had been deteriorating from the age of about 10. He suffers from retinitis pigmentosa. When registered blind he received basic cane training. He has had a guide dog for the last three years. At work he has a full time support worker and so is often guided. Subject $\mathrm{H}$ has not visited the evaluation area before.

All three subjects had the system and the testing arrangements fully explained to them before they agreed to take part. 


\subsubsection{Testing}

At the start of each session, subjects were asked their age, their sight history, the mobility training they had received, and how they generally got about. Subjects were then taken into room 302, which was designated the training room in the VE. Subject A had been in this room often, and in the room 339 opposite. Both he and subject $E$ were aware of the short corridor leading from the door from the stairs to the rest of the space. Subject A has visited two other offices down the side of the space, and had sat on one of the sofas. This was the limit of his knowledge. Subject $\mathrm{E}$ had been guided to an office at the end of the line of offices on two or three occasions, and this was the limit of her knowledge. Subject $\mathrm{H}$ had the short entrance corridor described to him for orientation. Figure 4 shows views of the space.

Figure 3 about here

Each subject was told that they were to use the Wii controls to explore a virtual representation of the open area, which contained some items of furniture and other items, and was edged by numbered offices and other doors. They were instructed in the use of the controls and the feedback they should expect, which were also demonstrated. They were given feedback on their use of the controls. They were asked to use the controls to explore virtual room 302 to get used to the controls and to ask any questions. The height of the infra red bar was adjusted to suit their comfortable holding of the controls. This initial phase took about 10 minutes per subject. Once they seemed familiar with the controls they were asked to move around the virtual room and find the virtual door of the room, to exit 
through it (this was the only door they could pass through), and then to use the controls to move around the virtual open space for as long as they liked. During their exploration, further feedback on their use of the controls was given, and, in the case of subjects E and H, they were given tasks to complete (e.g., "there are some tables, try and find them") to ensure they had explored all of the space. Exploration of the virtual space took around 20 minutes each. Once they had explored the virtual space to their and to the testers' satisfaction they were asked to return to virtual room 302. They were then asked to describe the space. The subjects were then asked to go out into the real space with their canes. Subjects A and E were told there was an object on table 2 and were asked to find it and take it to the fire door. Subject $\mathrm{H}$ was not very successful in exploring the virtual space so he was taken to the top of the real open space, asked to find the tables and from there the fire door.

All subjects were then asked some questions about the ease of use of the system and their views of it.

\subsubsection{Results}

Both subjects A and E managed to explore the whole of the virtual space, find all the objects in it and most of the doors. When asked to describe the space they gave a fairly accurate, spatial, description of it, its shape and the positions of the objects in it. For example, they managed to describe the spatial arrangement of the sofas and the tables with respect to them. When asked to find the object and take it to the fire door, they both did so directly and with confidence. They found the controls a bit challenging. They thought it would be a good way for people who are blind to learn to navigate new spaces. 
Subject H struggled to explore the space. Although he seemed to understand the controls and the feedback, he often went out of plane and collided with objects. In these cases he made little attempt to either get back in plane or out of collision. After a short while he was reminded what the feedback was telling him and what to do about it. He did manage to visit most of the space, but mainly by luck and prompting rather than by making a systematic effort to do so. He could not describe the space apart from saying he knew there was a space, he had no idea of the shape, he knew there were sofas, tables and bins but not where they were. He was not asked to find the object but taken into the space and asked where the tables were, which he found quite easily, and from there to go to the fire door. He went to it almost directly. While he had found the exploration difficult and did not seem to have much idea of the space, he did not have any trouble finding objects in the real space. His residual vision helped him find the tables, because the tables are light coloured, but this would not have helped him find the fire door from the tables.

Subjects A and E had some difficulty turning. Often they did not roll the Nunchuk far enough to effect a turn, and they over-rolled it when returning to centre. However, they both liked the correspondence between the movement of the device and movement effected in the VE. Subject H had the same problem, and reported the controls as difficult. He was reluctant to move the Wiimote at all.

\section{CONCLUSIONS}

Overall, the results of the evaluation were positive. Subjects A and E enjoyed using the system, appeared to develop spatial maps of the space, and were able to successfully 
navigate in a space which was largely new to them. Subject $\mathrm{H}$ was not so successful, but did seem to develop an idea of the layout. Subjects A and E both use computers regularly and picked up the controls very quickly. Subject $\mathrm{H}$ does use computers but described himself as a technophobe. He is often guided. Subject A often travels independently. Subject E is generally independent. Subject A is obviously familiar with the controls. Subjects E and H had both used the Wii game system previously. All three subjects developed an idea of the spatial layout of the real space, and were able to navigate in it. The results strongly support the idea that the system can be used to facilitate and support the construction of cognitive spatial maps and navigational strategies.

It is often noted in the literature that there is a wide range of strategies and navigational abilities used in spatial tasks amongst people who are blind (e.g., Hill et al, 1993). It was clear from the evaluation that training for subject $\mathrm{H}$ needed to be taken more slowly, and this may well be the case for other people. The present study was carried out very flexibly, as the system had never been used before; however, it is clear that more training should be given, and a more comprehensive training schedule should be devised. Use of the thumbstick may be easier than rolling the Nunchuk, and this will be an available option in future designs.

The use of the Wii devices allows a greater range of navigational information than the force feedback joystick used by others (e.g., Lahav and Mioduser, 2005, 2008). The Wiimote can be used to scan around the environment to explore and to decide where to direct motion. 
Other aids to navigation are to be developed in future systems. Presently, on transition to a new space, a description of the space is available via a button press. It is planned to make this option available at any time. Incorporation of auditory signposts and intelligent tutors into the system are planned. At present, the name of an object at which the Wiimote is pointing is available with a button press. Additional information could be incorporated at this point. Auditory signposts at appropriate places, such as by doors to new spaces, that explain where the door leads and how to navigate within the new area would be useful. Virtual tutors could provide additional information. Virtual tutors could appear at points where the traveler gets stuck to advise on how to make progress. An intelligent agent, which gives context-dependant spoken warnings and advice, is being designed to support the virtual cane.

Further, more rigorous testing of the system is required. Additionally, while the system has been designed in a dynamic and interactive way, consistent with the principles of usercentred and Universal Design, it is important that, now the potential of the system has been established, more formal analysis of it is performed. The relative effectiveness of the different types of feedback, and the incorporation of other features (such as intelligent tutors, the Wii Fit board) will be analysed and the results applied to the design of the system to optimize its functionality (and see Sanchez et al, 2002; Eriksson and Gardenfors, 2004). The development of spatial representations, and the transfer of such information into navigational strategies used in the real world, will be investigated.

The WWii, using as it does mainstream and relatively cheap Wii technology, offers significant potential for the advancement of the Universal Design of Interactive Technology. 


\section{REFERENCES}

Battersby S J (2007), Serious Games and the Wii - a technical report, in Brown, D, \& Evett, L, (2007) Workshop on Serious Games, 27th BCS SGAI International Conference on Artificial Intelligence, AI-2007, Cambridge, England, December 2007

Battersby S J (2008), The Nintendo Wii controller as an adaptive assistive device - a technical report, HEA ICS Supporting Disabled Students through Games Workshop, Middlesborough, $4^{\text {th }}$ February 2008.

Battersby S J, Brown D J, Standen P J, Anderton N \& Harrison M (2004), Design, development and manufacture of novel assistive and adaptive technology devices, Proc. 5th Intl Conf. Disability, Virtual Reality \& Assoc. Tech. (ICDVRAT), Oxford, UK, 2004, pp. 1-8.

Brown D J, Shopland N, Battersby S, Lewis J \& Evett L J (2007), Can Serious Games engage the disengaged?, Proc. Euro. Conf. on Games-Based Learning, Paisley, Scotland, pp. 37-46.

Brown D J, Battersby S \& Shopland N (2005), Design and evaluation of a flexible travel training environment for use in a supported employment setting. Int. J. on Disability and Human Development, 4, 3, pp. 251-258.

Chion M (1994), Audio-Vision: Sound on Screen, English edition, edited and translated by Claudia Gorbman, New York: Columbia University Press

Cummins P A \& Rieser J J (2008), Strategies of maintaining dynamic spatial orientation when walking without vision, In Blindness and Brain Plasticity in Navigation and Object Perception (J J Rieser, D H Ashmead, F F Ebner and A L Corn, Eds), Lawrence Erlbaum Associates, New York, pp. 227-238.

Eriksson Y \& Gardenfors D (2004), Computer games for children with visual impairments, Proc. 5th Intl Conf. Disability, Virtual Reality \& Assoc. Tech. (ICDVRAT), Oxford, UK, 2004

Golledge R G, Klatzky R L \& Loomis J M (1996) Cognitive mapping and wayfinding by adults without vision, In The Construction of Cognitive Maps (J Portugali, Ed), Kluwer Academic Publishers, Netherlands.

Grammenos D \& Savidis A (2006), Unified design of Universally Accessible games (say what?), Gamasutra, Dec. $7^{\text {th }} 2006$,

http://www.gamasutra.com/features/20061207/grammenos_01.shtml, accessed 20/10/2008

Grammenos D, Savidis A \& Stephanidis C (2005), UA-Chess: A Universally Accessible Board Game. In Proceedings of the 3rd International Conference on Universal Access in Human-Computer Interaction, G. Salvendy (Ed.), Las Vegas, Nevada, USA, July 2005. Lawrence Erlbaum 
Hill E W, Rieser J J, Hill M, Halpin J \& Halpin R (1993), How persons with visual impairments explore novel spaces: strategies of good and poor performers, J. Vis. Imp. and Blindness, 87, 8, pp. 295-301

Lahav O \& Mioduser D (2005), Blind persons' acquisition of spatial cognitive mapping and orientation skills supported by virtual environment, Int. J. on Disability and Human Development, 4, 3, pp 231-237.

Lahav O \& Mioduser D (2008), Haptic-feedback support for cognitive mapping of unknown spaces by people who are blind, Int. J. Human-Computer Studies, 66, pp. 23-35.

Lannen T, Brown D J \& Powell H (2002), Control of virtual environments for young people with learning difficulties, Disability and Rehabilitation, 24, pp. 578 - 586

Loomis J M \& Klatzky R L (2008), Functional equivalence of spatial representations from vision, touch, and hearing: relevance for sensory substitution, In Blindness and Brain Plasticity in Navigation and Object Perception (J J Rieser, D H Ashmead, F F Ebner \& A L Corn, Eds), Lawrence Erlbaum Associates, New York, pp. 155-185.

McCrindle R J \& Symons D (2000) Audio space invaders, Proc. $3^{\text {rd }}$ Intl Conf. Disability, Virtual Reality \& Assoc. Tech. (ICDVRAT), Alghero, Italy, 2000

Peek, B. (2008) Managed library for Nintendo's Wiimote: A library for using a Nintendo Wii Remote (Wiimote) from .NET, CodePlex, http://www.codeplex.com/WiimoteLib, accessed 1.2.08

Petrie H L, Penn P R, Kornbrot D, Furner S \& Hardwick A (2000) Haptic virtual environments for blind people: further explorations with the Phantom device, Proc. $3^{\text {rd }}$ Intl Conf. Disability, Virtual Reality \& Assoc. Tech. (ICDVRAT), Alghero, Italy, 2000

PIN (2005) Terraformers - Award Winning 3D Game also playable by blind, PIN Interactive, http://www.terraformers.nu/index.php?option=com_content\&task=view\&id=34\&Itemid=2 $\underline{9}$ accessed 29.3.09

Sanchez J, Jorquera L, Munoz E \& Valenzuela E (2002), VirtualAurea: perception through spatialized sound, Proc. $4^{\text {th }}$ Intl Conf. Disability, Virtual Reality \& Assoc. Tech. (ICDVRAT), Veszprem, Hungary, 2002

Simonnet M, Guinard J-Y \& Tisseau J (2006), Preliminary work for vocal and haptic navigation software for blind sailors, Proc. $6^{\text {th }}$ Intl Conf. Disability, Virtual Reality \& Assoc. Tech. (ICDVRAT), Esbjerg, Denmark, 2006, pp. 255-262.

Westin T (2004), Game accessibility case study: Terraformers - a real-time 3D graphic game, Proc. 5th Intl Conf. Disability, Virtual Reality \& Assoc. Tech. (ICDVRAT), Oxford, UK, 2004, pp. 95-100. 
Figures 


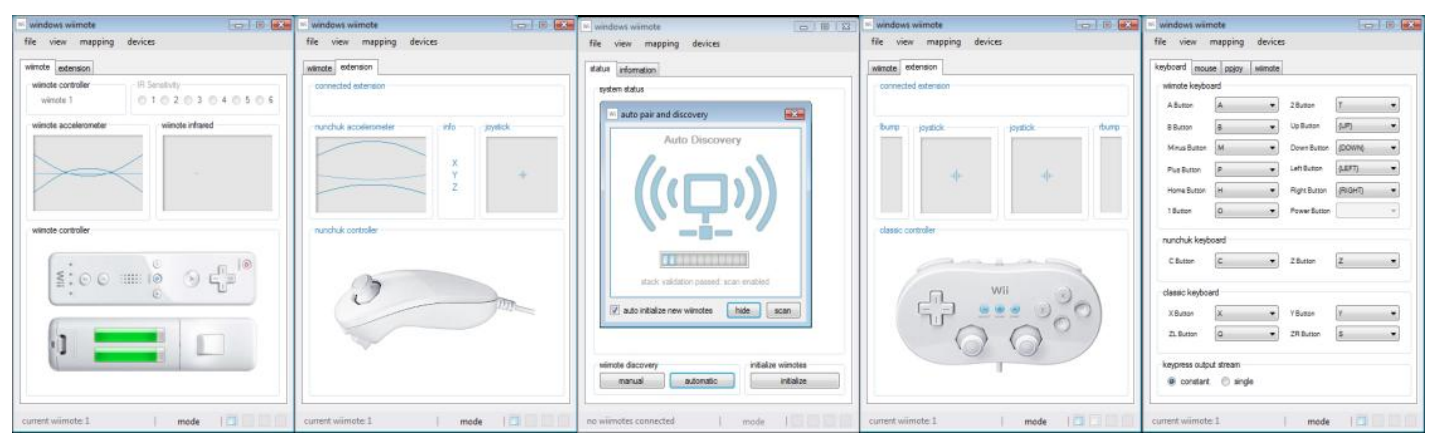

Figure 1: Part of the Windows Wii driver application interface

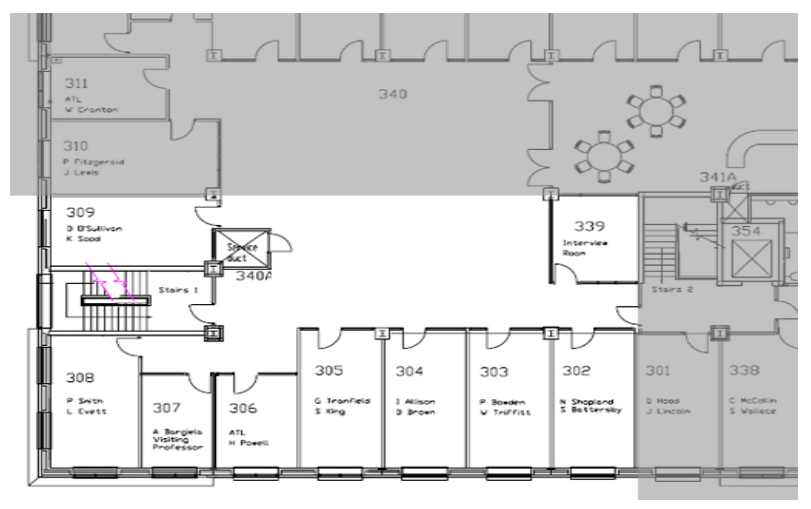

Figure 2: Floor plan - only the shaded section was used for navigation in the present evaluation
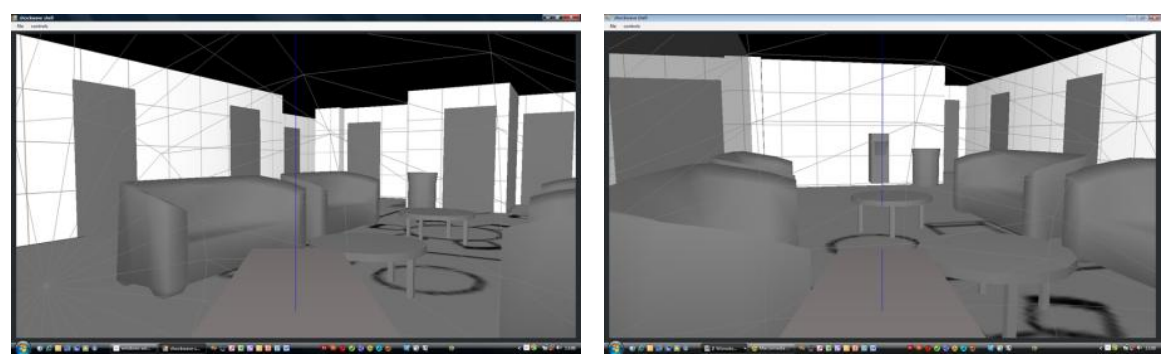

Figure 3: A view of the space from either end 